\title{
On the Role of Higher-Order Evanescent Modes in End-Offset Inlet and End-Centered Outlet Elliptical Flow-Reversal Chamber Mufflers
}

\author{
A. Mimani and M. L. Munjal \\ Facility for Research in Technical Acoustics, Department of Mechanical Engineering, Indian Institute of Science, \\ Bangalore 560 012, India
}

(Received 21 October 2010; Accepted 11 January 2012)

This paper deals with the role of the higher-order evanescent modes generated at the area discontinuities in the acoustic attenuation characteristics of an elliptical end-chamber muffler with an end-offset inlet and end-centered outlet. It has been observed that with an increase in length, the muffler undergoes a transition from being acoustically short to acoustically long. Short end chambers and long end chambers are characterized by transverse plane waves and axial plane waves, respectively, in the low-frequency range. The nondimensional frequency limit $k_{0}\left(D_{1} / 2\right)$ or $k_{0} R_{0}$ as well as the chamber length to inlet/outlet pipe diameter ratio, i.e., $L / d_{0}$, up to which the muffler behaves like a short chamber-and the corresponding limit beyond which the muffler is acoustically long are determined. The limits between which neither the transverse plane-wave model nor the conventional axial plane-wave model gives a satisfactory prediction have also been determined, the region being called the intermediate range. The end-correction expression for this muffler configuration in the acoustically long limit has been obtained using 3-D FEA carried on commercial software, covering most of the dimension range used in the design exercise. Development of a method of combining the transverse plane wave model with the axial plane wave model using the impedance $[\mathbf{Z}]$ matrix is another noteworthy contribution of this work.

\section{INTRODUCTION}

Determination of the acoustic attenuation performance of a muffler is essentially a 3-D problem inasmuch as it involves consideration of higher-order mode effects as well as 1-D axial plane-wave mode. The use of 1-D axial plane-wave analysis, which neglects the effects of higher-order mode effect altogether, has been used very widely ${ }^{1,2}$ with reasonably satisfactory results within the cut-off frequency. However, as one approaches the cut-on frequency of the first higher-order mode, deviations from plane-wave assumption start showing up, as demonstrated by Selamet and Radavich ${ }^{3}$ for concentric expansion chamber mufflers. It is thus shown that the diameter of the expansion chamber at the simple discontinuity is not the only parameter determining the role of higher-order modes in the transmission loss (TL) predictions. A resonance behavior similar to a quarter-wave resonator is thus seen in the cases when the $L / D$ ratio is low, indicating a dominant transverse or diametral wave propagation. It is argued that the length of the expansion chamber is not long enough for the evanescent modes to decay completely, and the higher-order modes thus completely dominate the axial plane-wave mode for such axially short chamber configurations.

A very similar result is also seen in the work of Kang and $\mathrm{Ji}^{4}$ wherein it has been reported that the end-correction technique is applicable only when the axial length of the expansion chamber is sufficiently long for the higher-order evanescent modes to decay sufficiently. They have even quantified the acoustic shortness in terms of the ratio of the axial length to the diameter of the expansion chamber, stating that the end corrections are not applicable for $L / D<0.3$, wherein the extended inlet expansion chamber is acoustically short. The end correction for a closed cylindrical side branch tube with consideration to the finite length effects on the acoustic end corrections has also been studied. ${ }^{5}$

In fact, there has been considerable 3-D analytical research to determine the acoustic attenuation performance of circular expansion chambers with or without extended inlet/outlet. ${ }^{6-9}$ There has also been 3-D analytical research on the circularshaped flow reversal chamber muffler, using either a modematching technique ${ }^{10}$ or superposition of velocity potentials. ${ }^{11}$ Denia et al. ${ }^{12}$ investigated the problem of elliptical chamber mufflers, analytically using the point superposition and mode superposition techniques involving the solution of Mathieu equations. Young and Crocker ${ }^{13}$ also investigated the performance of the elliptical flow-reversing mufflers, using 2-D finite element analysis (FEA). Selamet and Denia ${ }^{14}$ used 3-D FEA to analyze short elliptical chamber mufflers. However, the aforesaid studies are computationally more expensive as compared to the corresponding plane-wave analysis.

Recently, Mimani and Munjal ${ }^{15,16}$ analyzed axially short length mufflers of elliptical or circular shape by considering a 1-D transverse plane-wave model. In the former study, the matrizant method was used to solve the 1-D acoustic wave equation, while in the latter study, an analytical solution of the pressure field was obtained based upon the Frobenius solution. It was shown that for such short chambers, the transverse plane-wave model could satisfactorily predict the acoustic attenuation characteristics up to frequencies that are slightly higher than the cut-on frequency of the first higher-order mode. Mimani and Munjal ${ }^{15}$ also argued that as the axial length of the chamber was increased, the transverse plane-wave model 